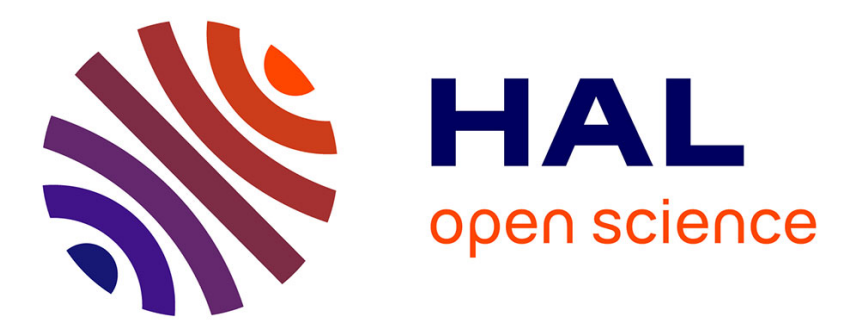

\title{
Nonlinear dynamics of turbulence driven magnetic islands. II. Numerical simulations
}

O. Agullo, M. Muraglia, S. Benkadda, A. Poyé, N. Dubuit, X. Garbet, A. Sen

\section{To cite this version:}

O. Agullo, M. Muraglia, S. Benkadda, A. Poyé, N. Dubuit, et al.. Nonlinear dynamics of turbulence driven magnetic islands. II. Numerical simulations. Physics of Plasmas, 2017, 24, pp.42309 - 42309. hal-01793877

\section{HAL Id: hal-01793877 \\ https://hal.science/hal-01793877}

Submitted on 17 May 2018

HAL is a multi-disciplinary open access archive for the deposit and dissemination of scientific research documents, whether they are published or not. The documents may come from teaching and research institutions in France or abroad, or from public or private research centers.
L'archive ouverte pluridisciplinaire HAL, est destinée au dépôt et à la diffusion de documents scientifiques de niveau recherche, publiés ou non, émanant des établissements d'enseignement et de recherche français ou étrangers, des laboratoires publics ou privés. 


\section{Nonlinear dynamics of turbulence driven magnetic islands. II. Numerical simulations}

O. Agullo, M. Muraglia, S. Benkadda, A. Poyé, N. Dubuit, X. Garbet, and A. Sen

Citation: Physics of Plasmas 24, 042309 (2017); doi: 10.1063/1.4981230

View online: http://dx.doi.org/10.1063/1.4981230

View Table of Contents: http://aip.scitation.org/toc/php/24/4

Published by the American Institute of Physics

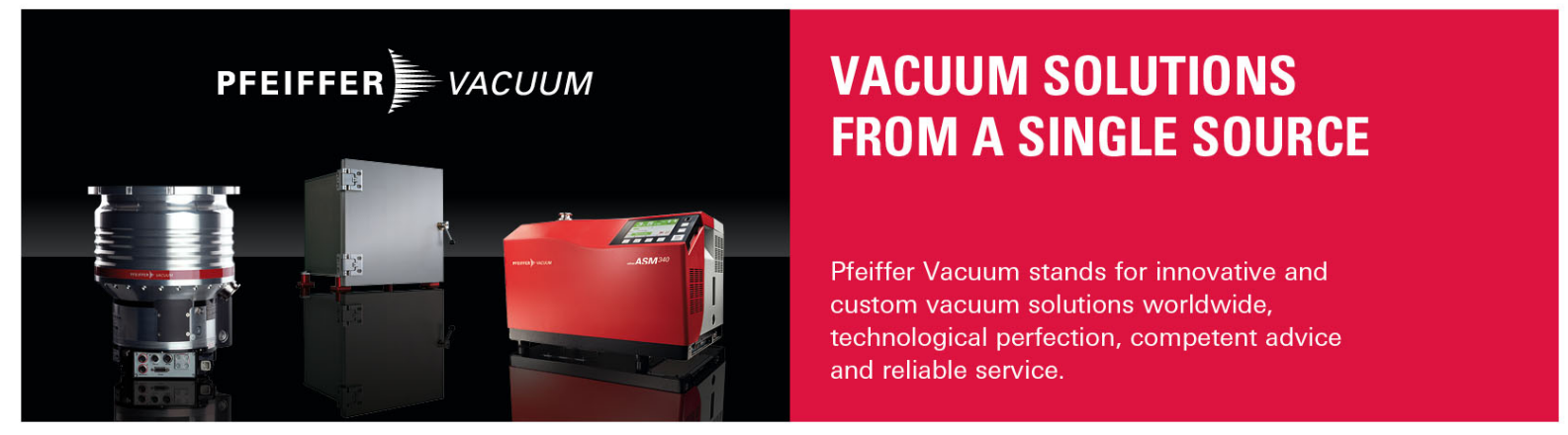




\title{
Nonlinear dynamics of turbulence driven magnetic islands. II. Numerical simulations
}

\author{
O. Agullo, ${ }^{1,2}$ M. Muraglia, ${ }^{1,2}$ S. Benkadda, ${ }^{1,2}$ A. Poyé, ${ }^{3}$ N. Dubuit, ${ }^{1,2}$ X. Garbet, ${ }^{4}$ and A. Sen $^{5}$ \\ ${ }^{1}$ Aix-Marseille Université, CNRS, PIIM UMR 7345, Marseille, France \\ ${ }^{2}$ France-Japan Magnetic Fusion Laboratory, LIA 336 CNRS, Marseille, France \\ ${ }^{3}$ Laboratoire de Physique, École Normale Supérieure de Lyon, CNRS Université de Lyon, Lyon, France \\ ${ }^{4}$ IRFM, CEA, 13108 St-Paul-Lez-Durance, France \\ ${ }^{5}$ Institute for Plasma Research, Bhat, Gandhinagar 382428, India
}

(Received 15 December 2016; accepted 31 March 2017; published online 21 April 2017)

The nonlinear dynamics of a turbulence driven magnetic island (TDMI) is investigated numerically in a reduced magnetohydrodynamic fluid model. The significance of identifying a characteristic signature of a TDMI for its experimental observation is discussed. The principal focus of our simulations is on the nature of the pressure profile flattening inside a TDMI, and we show that, in agreement with analytical predictions, a partial flattening occurs when the island size exceeds a critical value that is a function of the small scale interchange dynamics. We also present a model and test it numerically, which links explicitly the interchange turbulence and the island pressure flattening. Published by AIP Publishing. [http://dx.doi.org/10.1063/1.4981230]

\section{INTRODUCTION}

The dynamics of turbulence driven magnetic islands (TDMIs) has been studied numerically in a number of previous works ${ }^{1-4}$ because turbulence is suspected to be a trigger of seed islands and therefore of neoclassical tearing modes in tokamaks. In Ref. 5, a model has been developed to evaluate the role of the turbulence in the generation of magnetic islands. The issue is both to obtain a better understanding of the impact of the interchange scales on the transport properties of TDMIs and to infer some signatures of TDMIs. Indeed, the latter is a challenging experimental issue as we will discuss below, and it is therefore important to propose some signatures which could be detected by present day diagnostics. The results in Ref. 5 evaluate the turbulent perpendicular diffusivity, show, and/or explain why there is partial pressure flattening inside TDMIs and under which conditions it occurs. It also elucidates the link between the turbulence level and the mean pressure gradient. The purpose of this paper is to validate numerically these findings.

This paper is organized as follows. In Section II, the present experimental limitations to assess the role of turbulence in the generation of seed islands and in the detection of the TDMI signatures are discussed. In Section III, we present the numerical setup. We investigate the drive by the small scale interchange modes for a nonlinear magnetic island, in the regime where there exists a clear separation of scales between the interchange mode and the magnetohydrodynamic (MHD) scales. The MHD modes are taken to be linearly stable with respect to the tearing and interchange instabilities. In Sec. IV, we focus on the origin of the selfsustainment of TDMI, and in Section V, we show the existence of a critical island size above which a partial pressure flattening of TDMI occurs. In Sec. VI, we study the link predicted in Ref. 5 between the turbulence generated in a radially localized interchange band (IB) and the mean pressure flattening throughout the magnetic island. Finally, in
Section VII, we present a discussion of our results and make some concluding remarks.

\section{EXPERIMENTAL LIMITATIONS AND OBSERVATIONS}

In simulations, the possibility that turbulence may trigger seed islands is becoming quite well established, including in the gyrokinetic framework. ${ }^{4}$ Nevertheless, to prove the existence of a TDMI, there is a necessity to show it experimentally. One may ask if this is feasible in terms of present experimental capabilities or possibly, if some explicit signatures of TDMI have already been observed. These aspects are usually not discussed, even though this is an important point to guide future research. From an experimental point of view, the detection of turbulence driven seed islands and the unequivocal identification of their origin are difficult tasks. The difficulty arises from the non-availability of efficient diagnostic tools that can clearly quantify the interaction between micro-turbulence and MHD modes. While Doppler reflectometry measurements allow the reconstruction of density spectra for typical scale lengths in the range of $0.1<k_{\perp} \rho_{s} \leq 20$, they do not cover the MHD scales. On the other hand, using ECE (electron cyclotron emission) or Mirnov oscillation measurements, one can obtain spectra between 10 and $80 \mathrm{kHz}$ with poloidal mode numbers $m \leq 8$ which corresponds to $k_{\perp} \rho_{s}=m \rho_{s} / r_{s}<0.01$. A typical island has $k_{\perp} \rho_{s} \approx 10^{-3}$, and the seed islands span an intermediate range of scale lengths, roughly $k_{\perp} \rho_{s}$ of the order of or smaller than 0.1 . When they are larger, the bootstrap current will produce an amplification. When they are smaller, it is difficult to distinguish them from the natural magnetic fluctuations due to turbulence. Moreover, the observation of a spectral magnetic structure on intermediate scales would not prove that it is a turbulence driven magnetic island. Indeed, the key diagnostic evidence for that would be to demonstrate that there is a strong mode coupling (or a chain of couplings) 
between the island and the turbulence. However, because of diagnostics limitations, there are very few experimental studies in tokamaks that address this problem. Most observations are limited to MHD mode coupling phenomena using bispectrum analysis. ${ }^{6}$ An exception to this trend is the bi-coherence analysis studies carried out on the Brazilian tokamak TCABR that shows a strong correlation between MHD activity and electrostatic turbulence driven by Mirnov oscillations. ${ }^{7}$ However, the electrostatic signal was obtained by inserting electrostatic probes close to the boundary between the edge and the scrape-off layer. The MHD activity was not linked to hot core plasma physics and therefore to the generation of $(2,1)$ or $(3,2)$ magnetic islands.

The above observations underscore the point that it is necessary to have knowledge of some specific signatures associated with TDMIs in order to experimentally detect them and establish their existence. At the present time, diagnostics performing island detection and/or the reconstruction of the q profile-which is required to determine if there is a tearing instability - do so by measuring quantities which are radially averaged over a typical length scale of the order of a centimeter. In most tokamaks, an island of this size would satisfy the relation $w>w_{c}^{\text {seed }}$ which is the threshold condition necessary for them to be amplified by the loss of the bootstrap current, ${ }^{8}$ and therefore, they cease to be seed islands. Thus, these diagnostics are incapable of addressing the question of the generation of seed islands. Measurements of island pressure flattening constitute another efficient way of detecting islands whose widths are such that $w \gg w_{c}^{\text {flat }}$. $w_{c}^{\text {flat }}$ is the critical size above which a pressure flattening of the pressure inside the island occurs. It is proportional to the fourth power of the ratio of perpendicular and parallel diffusivities $w_{c}^{\text {flat }} \propto\left(D_{\perp} / D_{\|}\right)^{1 / 4}$. 9 The pressure is equalized over the whole island if the sources (or sinks) of both temperature and density inside the island can be neglected. We will see that, in the presence of small scale instabilities or turbulence, one should consider the presence of a source. As discussed above, when the bootstrap current is negligible, a TDMI presents a specific signature, namely, a partial pressure flattening that can possibly be detected experimentally. The origin of a TDMI is the small-scale turbulence inside the island or in its vicinity. The measurement of partial pressure flattening in such an island implies that its width satisfies the condition $w_{c}^{\text {flat }} \ll w \leq w_{c}^{\text {seed }}$.

Partial pressure flattening inside magnetic islands has already been observed in experiments. ${ }^{10-14}$ In fact, when a source perturbs or creates a magnetic island, a localised and persistent concentration of density into the magnetic island can be observed. In particular, it occurs when pellets are injected into JET tokamaks and generate density and impurity snakes. ${ }^{13}$ Peaked ion temperature profiles have also been observed inside islands in mode locking phases ${ }^{14}$ after a transition from the $\mathrm{H}$ mode to the L mode in JT-60U. The latter experiment also requires a source, more precisely, neutral beam injection, which can induce a strong rotation shear at the boundary of the magnetic island. Together with a significant reduction in the thermal diffusivity inside the island, it contributes to improving the confinement. Bifurcation of the transport properties inside magnetic islands, i.e., a strong reduction in the diffusivity, is also observed in the tokamak DIII-D where the magnetic island is induced by an external coil, in the LHD stellerator, ${ }^{15}$ and in the MST reverse field pinch device after a sawtooth event. ${ }^{16}$ The reduction in the diffusivity into the island can potentially be linked to a reduction in the turbulence level. In the discussed cases, either a source, a strong MHD dynamics, or an event which reduces the global confinement in the device seems to be at the origin of the enhanced confinement into the magnetic islands. In contrast, in the TEXT tokamak, ${ }^{10}$ a partial flattening of density was clearly observed in a regime where a strong interaction between the island and turbulence was at play. No physical explanation of this observation was given. Rather, it was attributed to limitations in the spatial resolution of the diagnostics. The hypothesis put forward in Ref. 10 for this observation was the presence of a strong turbulent diffusion in the vicinity of the $\mathrm{X}$ point. Indeed, one might expect $w_{c}^{\text {flat }}$ to be proportional to $\left(D_{\perp}^{\text {turb }} / D_{\|}\right)^{1 / 4}$, where $D_{\perp}^{\text {turb }}$ is the turbulent perpendicular diffusivity. Therefore, enhanced perpendicular diffusivity would enlarge the critical island width below which there is no flattening, which could potentially explain the observations. However, this partial flattening is, alternatively, possibly linked to the fact that small scale turbulence in the vicinity of the resonant surface where the island grows inhibits a complete flattening ${ }^{23}$ when the condition $w \gg w_{c}^{\text {flat }}$ is satisfied. As we shall see, turbulence can, indeed, play the role of the source in the vicinity of the resonance. In that case, the predictions based on the ratio $w \gg w_{c}^{\text {flat } 9}$ are incomplete and one should consider the predictions presented in Ref. 5. In particular, the analytical link between the turbulence level and the mean pressure gradient inside an island could be an interesting path if one wants to detect TDMIs experimentally. Indeed, to some extent, it shows that the pressure gradient inside the island is linked to a power of the turbulent thermal and kinetic energies and is also inversely proportional to the size of the island. The measure of the impact of magnetic islands on turbulent fluctuations has been assessed experimentally recently. ${ }^{17}$ One may therefore expect to evaluate the converse situation where by modifying the turbulence level, one could observe a modification of the island transport properties such as the pressure flattening. Before addressing through numerical simulations the role of the turbulence and the turbulent diffusivity in the pressure flattening of TDMI, we describe below the model we shall use. Let us emphasize that the critical island width for pressure flattening that we will consider, in the following, will be linked to the level of the turbulence. To specify it, we will note this width $w_{c}^{\text {turb }}\left(w_{c}^{\text {turb }} \equiv w_{c}^{\text {flat }}\right)$.

\section{NUMERICAL SETUP}

To study the dynamics of TDMIs, we have used the model presented in Refs. 18 and 19, which considers the time evolution of the electric potential $\phi$, the electronic pressure fluctuations $p$, and the total magnetic flux $\psi=\psi_{\mathrm{eq}}+\tilde{\psi}$

$$
\frac{\partial}{\partial t} \nabla_{\perp}^{2} \phi+\left\{\phi, \nabla_{\perp}^{2} \phi\right\}=\left\{\psi, \nabla_{\perp}^{2} \psi\right\}-\kappa_{1} \frac{\partial p}{\partial y}+\mu \nabla_{\perp}^{4} \phi,
$$




$$
\begin{gathered}
\frac{\partial}{\partial t} \psi=\{\psi, \phi-p\}-v_{\star} \frac{\partial \psi}{\partial y}+\eta \nabla_{\perp}^{2} \tilde{\psi}, \\
\frac{\partial}{\partial t} p+\{\phi, p\}=-v_{\star}\left(\left(1-\kappa_{2}\right) \frac{\partial \phi}{\partial y}+\kappa_{2} \frac{\partial p}{\partial y}\right) \\
+\hat{\rho}^{2}\left\{\psi, \nabla_{\perp}^{2} \psi\right\}+\chi_{\perp} \nabla_{\perp}^{2} p .
\end{gathered}
$$

Eqs. (1)-(3) are normalized using the characteristic Alfvèn speed $v_{A}$, the magnetic shear length $L_{\perp}$, and the Alfven time $\tau_{A}=L_{\perp} / v_{A}$. A semi-spectral code called $\mathrm{AMON}^{3}$ is used including a $2 / 3$ dealiasing rule in the $y$ (poloidal) direction with 256 poloidal modes and a resolution of 1025 grid points in the $x$ (radial) direction. The computational box size is $L_{x}=2 \pi$ and $L_{y}=5 \pi$. The perturbed fields are periodic in the $y$ direction and are set to zero at the radial boundaries. The resistivity and the perpendicular diffusion $\left(\eta, \chi_{\perp}\right)$ are taken to be equal. The viscosity and the normalized Larmor radius are set equal, respectively, to $\mu=10^{-4}$ and $\hat{\rho}=0.058$. This simplified model takes into account interchange and tearing instabilities and as a consequence is very useful to study the large island generation mechanism involving smallscale interchange modes. More precisely, using the Fourier decomposition of the fields which is typically defined as $\psi(x, y, t)=\sum_{m \in \mathbb{Z}} \psi_{m}(x, t) \times \exp \left(i k_{m} y\right)$, one can identify the nature of a given mode $m$ thanks to the parity of the eigenfunctions of the three fields: the resistive interchange mode $m$ has odd, even, and even parities with respect to $x \in$ $\left[-L_{x} / 2, L_{x} / 2\right]$ for $\left(\psi_{m}(x, t), \phi_{m}(x, t), p_{m}(x, t)\right)$, respectively, and even, odd, and odd parities for a tearing mode. In this model, the magnetic equilibrium is given by the Harris current sheet model, namely, $\mathbf{B}_{\mathrm{eq}}(x)=\tanh \left(\frac{x}{a}\right) \hat{y} \cdot a$ determines the width of the profile and therefore, for a fixed size of the box, the value of the tearing mode stability index parameter $\Delta^{\prime}$. In order to investigate the regime where a large magnetic island is driven by the interchange modes, all the modes are taken to be stable with respect to the tearing instability. ${ }^{20-22}$ In particular, we have set $\Delta^{\prime}=-0.45$ for the mode $m=1$ : linearly, there is no magnetic island. The interchange instability is controlled by the constant equilibrium pressure

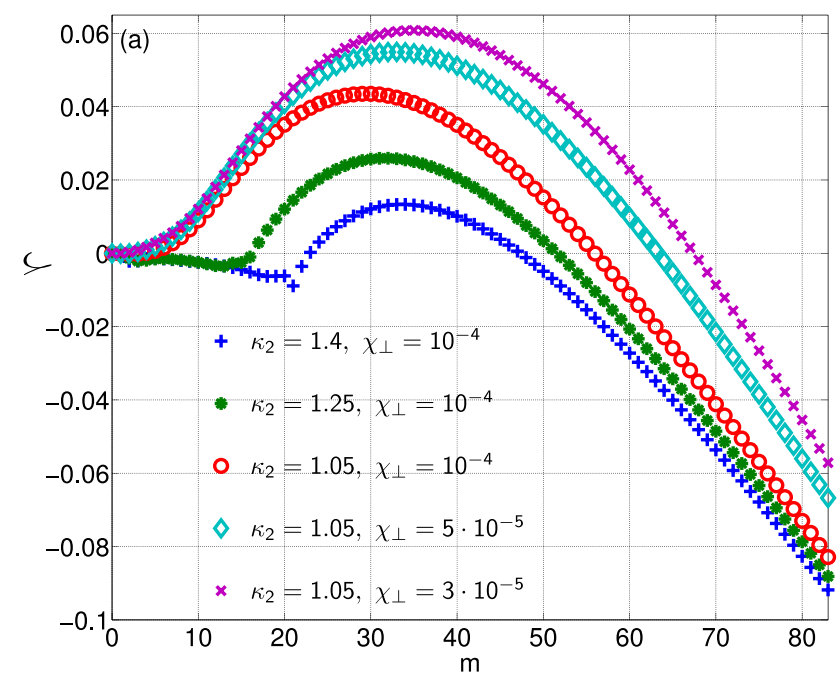

gradient fixed by the diamagnetic velocity $v_{\star}=-0.01$ and the curvature parameter $\kappa_{1}=10$.

Simulation parameters are chosen such that the small scale modes are linearly unstable with respect to the interchange instability. In contrast to Ref. 2 where large scales were weakly unstable with respect to the interchange mode, we fix the parameters such that the largest scales are linearly stable. Compared with the previous works where the flattening of TDMI was observed, ${ }^{23}$ we also enlarge the scale separation between the island and interchange scales. One can ask whether the beating mechanism of the generation of TDMI described in Ref. 2 is still relevant in the present set up? In order to answer this question, we have performed new simulations for different values of the interchange parameter $\kappa_{2}=[1.05,1.25,1.4]$ and the diffusion coefficient $\chi_{\perp}=\left[10^{-4}, 5 \times 10^{-5}, 3 \times 10^{-5}\right]$. The corresponding linear spectra are plotted in Fig. 1[left]. Although large scale modes, including the $m=1$ mode, present an interchange parity, they are stable (negative linear growth rate) in these new simulations. Moreover, the most interchange unstable mode is about $m_{\star} \sim 34$, i.e., the linear setup is characterized by a realistic ratio between (seed) islands and interchange mode numbers.

Fig. 2 shows the time evolution of the magnetic energy of the main modes of the dynamics $m=0, m=1, m=34$, and $m=35$ for a nonlinear run with $\kappa_{2}=1.4$ and $\chi_{\perp}=10^{-4}$. It shows the nonlinear generation of a TDMI. It confirms the results obtained in Ref. 2 and shows that, both scale separation and stability of the large scale do not impact the nature of the mechanism generating a TDMI: an island is generated in the quasi-linear phase and has a growth rate equal to $2 \gamma_{\star}$. More precisely, the dynamics can be decomposed into three stages. For $0 \leq t / \tau_{A} \leq 400$, the linear regime is recovered and the mode growth rates are in agreement with the linear spectra shown in Fig. 1(left): small scales modes are unstable with a growth rate $\gamma_{34} \sim \gamma_{35}=\gamma_{\star}=1.3 \times 10^{-2}$ and large scale modes $(m=0$ and $m=1)$ are stable and present an interchange parity. There is no linear generation of magnetic islands. Then, for $400 \leq t / \tau_{A} \leq 1000$, a quasi-linear regime

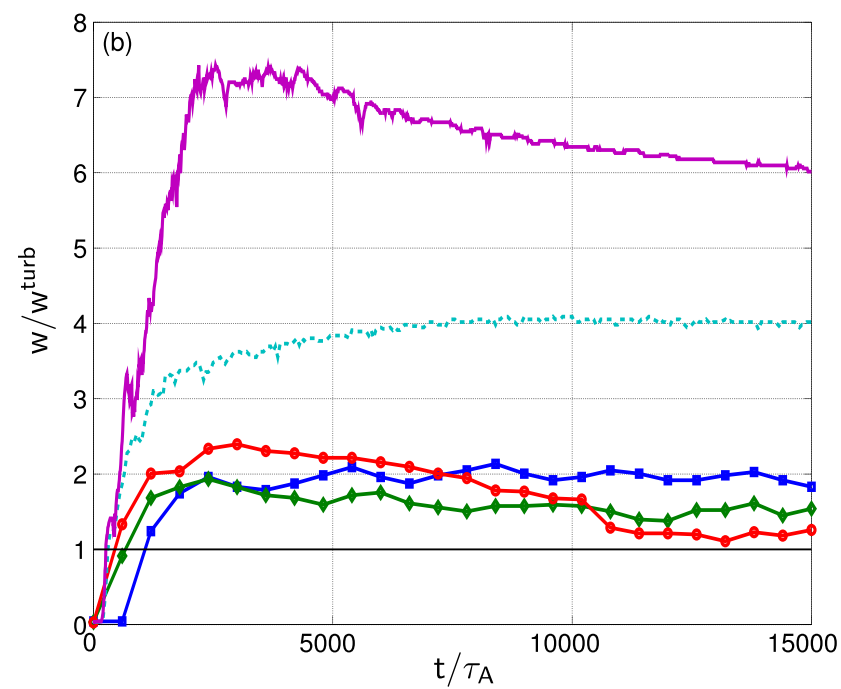

FIG. 1. [Left] Linear spectra: growth rate $\gamma$ as a function of the poloidal mode number $m$. [Right] Time evolution of the island size $w$ normalized to the turbulent critical island size $w_{c}^{t u r b}$. For each color, the run parameters are the same in the left and right graphs. 


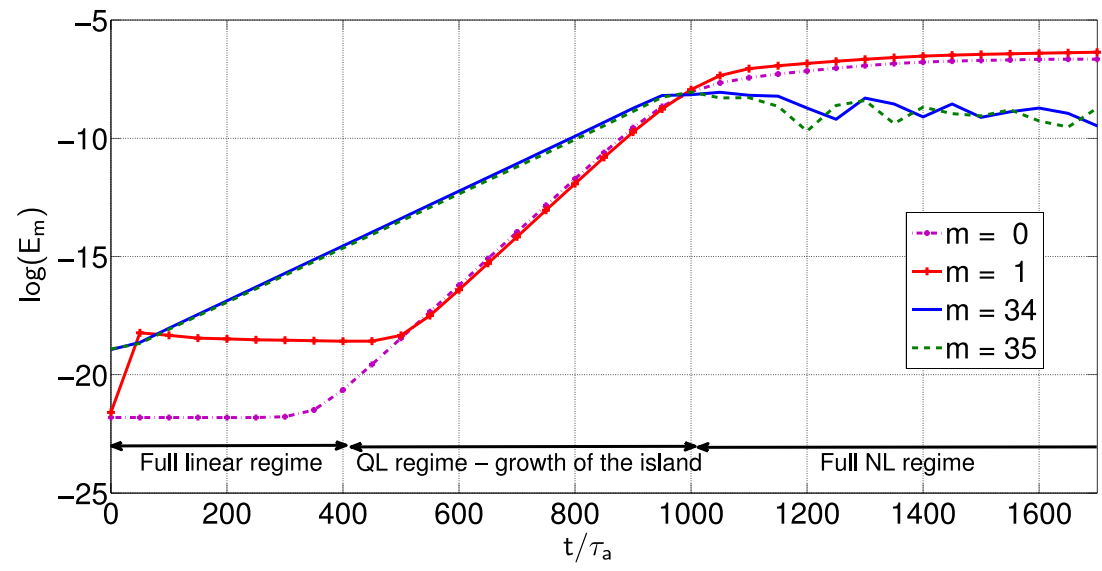

FIG. 2. $\kappa_{2}=1.4$ and $\chi_{\perp}=10^{-4}$. Time evolution of the magnetic energy of the main modes of the nonlinear simulation $m=0,1,34,35$. arises. Large scale modes $(m=0$ and $m=1)$ become unstable with a linear growth rate given by $\gamma_{0}^{N L} \sim \gamma_{1}^{N L} \sim 2 \gamma_{\star}$. The radial dependence of the large scale eigenfunction $\psi_{1}(x)$ adopts a tearing parity in the quasilinear regime. The island does not disappear in the nonlinear regime $t / \tau_{A}>1000$. The nonlinear dynamics generating a TDMI that we have just described is not specific to the run presented in Fig. 2. The linear spectra we have used to perform nonlinear simulations (see Fig. 1) exhibit various ranges of unstable modes, including cases where there is a clear scale separation between the MHD and interchange scales. In essence, we have found that the generation of a TDMI does not depend on the precise shape of the spectra. However, the spectra determine the values of the critical turbulent island size. We will show in Section $\mathrm{V}$ the latter impact on the asymptotic properties of the dynamics and the pressure profile flattening of the magnetic islands.

\section{SELF-SUSTAINMENT OF TDMI}

One may wonder at this point about the basic mechanism underlying the self-sustainment of the magnetic structure and whether it differs from the one at play for the tearing instability. The magnetic equilibrium and the $\Delta^{\prime}$ parameter fix the condition for the tearing instability to exist and the amount of free energy available, and the resistivity value determines to some extent the reconnection rates. However, the fundamental reason for which the magnetic structure is self-sustaining is the presence of a quadrupolar flow. Indeed, basically, a large fraction of flow is penetrating into the island transversely to the magnetic field lines in the vicinity of the $\mathrm{X}$ point. It is expelled from the island at the $\mathrm{O}$ point poloidal level, where radial island extension is maximal and magnetic tension is minimal. In particular, the acceleration of the outflow opposes the Lorentz forces, and the balance between the two determines the growth, the decrease, or the saturation of the flow structure. At the same time, such a flow structure advects magnetic flux through the $\mathbf{v} \times \mathbf{B}$ term and sustains the current sheet. The growth of the dominant harmonic of both the flow and the magnetic field, but not the higher harmonic contributions, determines finally the dynamics of the global flow-island structure and the island width. ${ }^{24}$ Let us emphasize, that more generally, whatever the alternative known mechanisms generate and amplify magnetic islands such as Sweet-Parker and Petschek models, the flow presents a quadrupolar structure. It turns out that this mechanism is still at play for TDMI. Indeed, Fig. 3 (left) presents the snapshot of the difference between the electrostatic potential and the pressure, which drives the advection of the flux in Ohm's law Eq. (2). As far as the system enters the quasilinear regime, breaking and reconnection of the magnetic field lines have occurred and a quadrupole $\phi-p$ structure goes along with the island growth. By advecting the magnetic flux, the quadrupolar structure sustains the growth of the island. In Fig. 3(right) where the snapshot of the magnetic flux $\psi$ during the nonlinear regime is drawn, the magnetic island structure is clearly observed. Thus, there is some universality in the presence of a quadrupole when an island is generated. The access to the full non-linear regime accelerates the island, and the quadripolar structure disappears gradually. It does not suppress the island. The asymptotic regime is characterized by a statistically stationary state for the fluctuations. In that case, the sustainment of the current sheet is not anymore provided by the inductive electric field but by the dynamo term alone $\left\{\phi_{\text {isl }}-p, \psi\right\}$ in the island frame. Here, $\phi_{\text {isl }}=\phi$ $-v_{\text {isl }} x$, and the island rotates poloidally at velocity $v_{\text {isl }}$.

\section{EXISTENCE OF A CRITICAL ISLAND SIZE FOR THE PARTIAL PRESSURE FLATTENING OF TDMI}

The critical island width above which a flattening of the pressure inside the island should occur has been evaluated to be $\mathrm{e}^{5,9}$
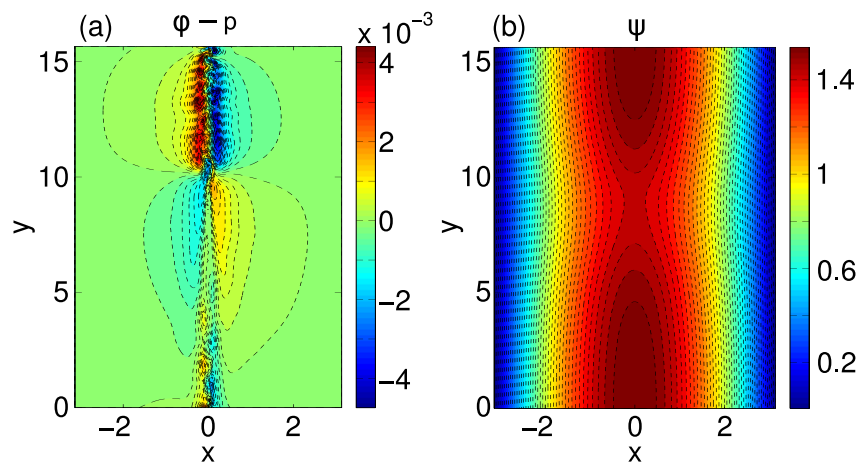

FIG. 3. $\kappa_{2}=1.05$ and $\chi_{\perp}=3 \times 10^{-5}$. Snapshots of the difference between the electrostatic potential $\phi$ and the pressure $p$ in the quasi-linear regime (left) and of the magnetic flux $\psi$ in the full nonlinear regime (right). 


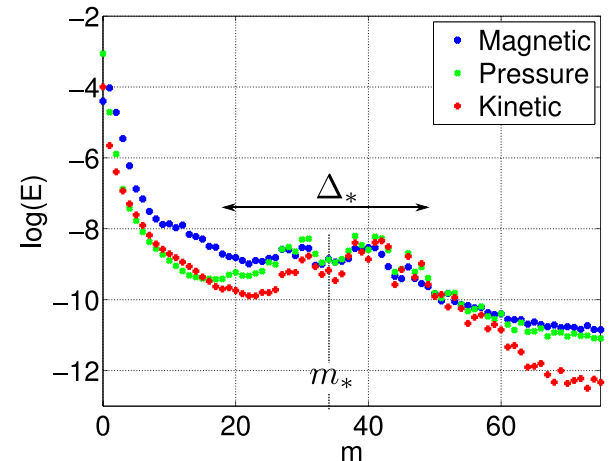

FIG. 4. Spectra in the nonlinear phase for the run $\kappa_{2}=1.05$ and $\chi_{\perp}=3 \times$ $10^{-5}$ at $t=7500 \tau_{A}$ for which $m_{\star}=34$ and $\Delta_{*}=15$.

$$
w_{c}^{\text {turb }}=\sqrt{8}\left(\chi_{\perp}^{\text {turb }} / \chi_{\|}\right)^{1 / 4} \sqrt{a / k_{y}} .
$$

$\chi_{\perp}^{\text {turb }} \approx \Delta_{\star} \gamma_{\star} / k_{\star}^{2}$ is the turbulent perpendicular diffusion coefficient. The implicit parallel diffusivity is given by $\chi_{\|}=\eta^{-1} \hat{\rho}^{2} . \Delta_{\star}$ is the spectral extension of the most unstable interchange modes which are characterized, in the nonlinear regime, by an equipartition between magnetic and pressure energy. We will specify it below. In this section, we check the validity of the prediction Eq. (4) and more generally the existence of a critical width for TDMIs above which pressure flattening occurs. Indeed, turbulence might not necessarily be reduced to a transport parameter at large scales for this question. Moreover, the hypothesis that $\Delta_{*}$ interchange modes enhance a large scale turbulent transport might not be true.

The parameter $\Delta_{*}$ is linked to a natural scale separation which appears in the nonlinear regime. To bring out this point, we present in Fig. 4 the spectra of the kinetic, pressure, and magnetic energies in the nonlinear regime. They present properties shared by all the runs of this paper, more generally which are valid for a wide range of parameters, as already emphasized in Ref. 5: First, large scales $m \leq 1$ dominate energetically. Second, intermediate scales are characterized by the equipartition between pressure and kinetic energy. They correspond to poloidal mode numbers in the range $3 \leq m<m_{\text {th. }}$ with $m_{\text {th. }}=17<m_{\star}$ in Fig. 4 . Fig. 5 shows the modes $m=8$ in real space, i.e., the sum of the mode $m= \pm 8$ in the complex space. It emphasizes that such equipartition is due to the adiabatic trend of the system at intermediate scales, except in the vicinity of the external part of the island $(x \approx w / 2)$ where $\phi_{8}$ and $p_{8}$ have the same sign. Third, there is a tendency to an equipartition between magnetic and pressure energy spectra for the dominant interchange scales $\left|m-m_{\star}\right| \leq \Delta_{*}$. The fields at those scales will be noted $\left(\phi^{\prime}, p^{\prime}, \psi^{\prime}\right)$.

Let us now check whether or not a critical island size $w_{c}^{\text {turb }}$ exists for the pressure flattening. In this work, the set of parameters has been chosen such as we can explore a regime where at saturation $w / w_{c}^{\text {turb }}$ ranges roughly from 0.5 to 3 (see Fig. 1(b)). It was obtained by varying the curvature parameter $\kappa_{2}$ and the dissipative parameter $\chi_{\perp}$ in order to modify both the turbulent diffusivity and the predicted critical island width $w_{c}^{\text {turb }}$. In a previous work, ${ }^{23}$ the growth of the island was such that $w \gg w_{c}^{\text {turb }}$ as soon as the system entered the quasilinear phase, and therefore, it was not possible to verify the existence of a critical width for TDMIs.

Fig. 1(b) explicitly shows the time evolution of the island size normalised to the critical turbulent island size $w_{c}^{\text {turb }}$. It turns out that the asymptotic size depends on the free energy interchange level. Indeed, the larger the dominant growth rates and/or $\chi_{\perp}^{\text {turb }}$, the larger the asymptotic island size. This is true only if $\chi_{\perp}^{\text {turb }}$ is large enough. Indeed, Fig. 1 also shows that, in the nonlinear asymptotic regime, the ratio $w / w_{c}^{\text {turb }}$ is not monotonic, below some threshold value of $\Delta_{*}^{-1} \chi_{\perp}^{c}$ turb $/ \chi_{\perp}$. This threshold is between 3 and 6 . When $\gamma_{\star}$ decreases, $\Delta_{*}^{-1} \chi_{\perp}^{\text {turb }} / \chi_{\perp}$ decreases from 10.3 to 0.7 , for the runs presented in Fig. 1. When $\chi_{\perp}^{\text {turb }} / \chi_{\perp}$ is below the critical
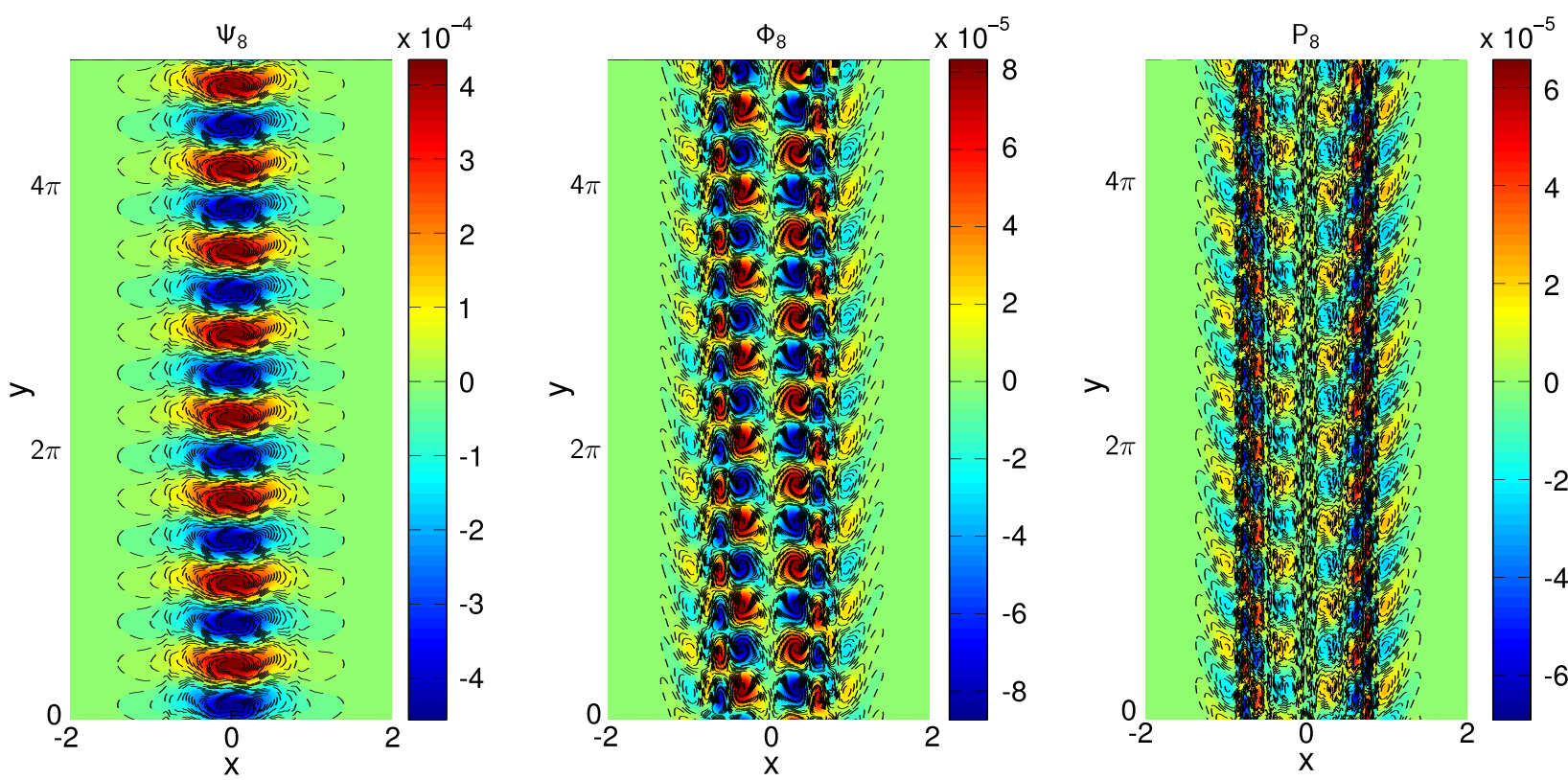

FIG. 5. Snapshot of the modes $m=8$ in real space in the nonlinear phase for the run $\kappa_{2}=1.05$ and $\chi_{\perp}=3 \times 10^{-5}$ at $t=7500 \tau_{A}$. Note that these modes have the tearing parity: $\left(\psi_{8}, \phi_{8}, p_{8}\right)$ are, respectively, even, odd, and odd with respect to $x$. 
value, the asymptotic island size is of the order of $w_{c}^{\text {turb }}$. More precisely, it fluctuates in the range $\left[w_{c}^{\text {turb }} / 2, w_{c}^{\text {turb }}\right]$ and seems to be roughly independent of $\chi_{\perp}^{\text {turb }}$. A natural question is whether or not, in this regime, the partial flattening of the island disappears.

In order to verify if partial flattening occurs only when $w$ becomes significantly greater than $w_{c}^{\text {turb }}$, we consider the nonlinear dynamics of runs with $\chi_{\perp}=10^{-4}$. Then, the asymptotic island sizes satisfy $w / w_{c}^{\text {turb }} \leq 1$. We find that, concomitantly, the radial pressure profiles of such weak turbulence level simulations are almost not flattened. It is shown in Fig. 6(left) for the case $\kappa_{2}=1.4$ and $\chi_{\perp}=10^{-4}$ (blue square lines). The time evolution of the normalized pressure gradient $-\left\langle d p_{0} / d x\right\rangle / v_{\star}$ is also shown in Fig. 6(right). $\left\langle d p_{0} / d x\right\rangle$ is a mean of $d p_{0} / d x$ inside the island. This ratio remains close to one, and therefore, there is almost no flattening when $w$ is slightly above or of the order of $w_{c}^{\text {turb }}$. Considering again the latter case, we observe that a very weak flattening, oscillating in time, exists. In fact, the island size oscillates at the same frequency, in phase opposition (see Fig. 1). This is in agreement with the expectation that the flattening increases with the island size.

A larger turbulence level regime can be analysed by decreasing the parameter values to $\kappa_{2}=1.05$ and $\chi_{\perp}=3$ $\times 10^{-5}$. As predicted, Fig. 1 shows that the island size is increased, reaching $w / w_{c}^{\text {turb }} \approx 3$ after the quasi-linear phase. As a consequence, the pressure flattening is intensified and/or the ratio $\delta p^{\prime} / v_{\star}$ fluctuates around 0.25 in this phase, see Fig. 6(right). Moreover, in the far nonlinear phase, there is a simultaneous decrease in the island width and the flattening of the pressure profile. The origin of the reduction of the island size is due to a weakening of the interchange power source. The latter is due to the flattening effect. The pressure profiles are also shown in Fig. 6(left). In contrast to the results with a higher $w / w_{c}^{\text {turb }}$ ratio, ${ }^{23}$ we observe a slow disappearance of the flattening inside the island.

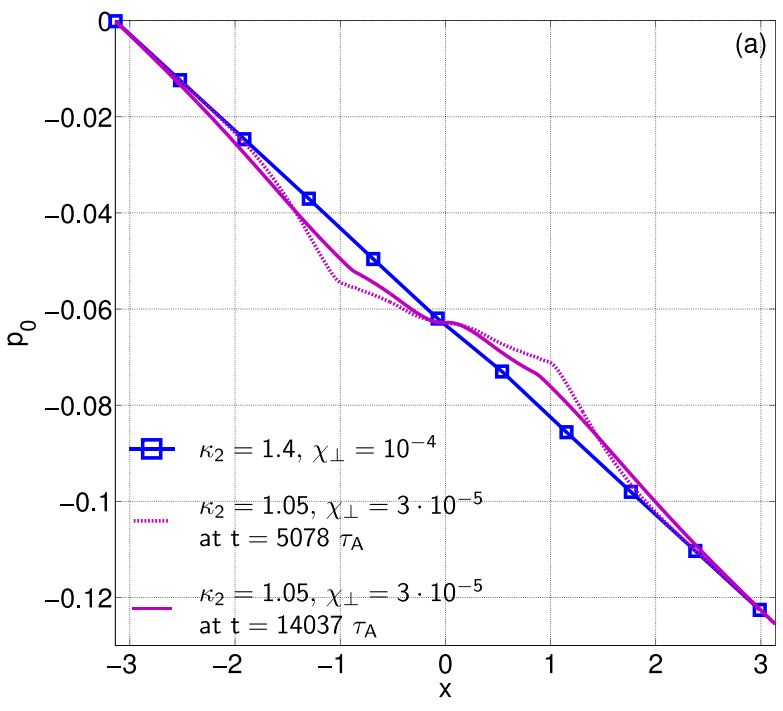

\section{AN EXPLICIT LINK BETWEEN THE TURBULENCE AND PRESSURE PROFILE INSIDE THE ISLAND}

In Ref. 5, in particular in Eq. (41), it has been proposed that the constant in the space mean pressure gradient of the magnetic island $\nabla P_{0}^{\text {isl }}$ is linked to the interchange small scale turbulence through the identity

$$
\begin{aligned}
\nabla P_{0}^{\mathrm{isl}}(t)= & -v_{\star}+\beta\left\langle\frac{2}{w} \int_{0}^{t} d t\right. \\
& \left.\left.\times\left\langle 2 \sum_{\left|m-m_{\star}\right| \leq \Delta_{*}, m>0} k_{m} \frac{d}{d x} \mathcal{I}\left(\hat{p}_{m} \hat{\phi}_{m}^{\star}\right)\right\rangle\right\rangle_{x}\right\rangle .
\end{aligned}
$$

The integrated term in brackets $\langle.\rangle_{x}$ corresponds to the interchange source term $\bar{S}_{\phi^{\prime}, p^{\prime}}$ introduced in Ref. 5 and results from the nonlinear transfer of energy from interchange scales to the island scales through the advection term $\{\phi, p\}$. $\hat{p}_{m}(x)$ and $\hat{\phi}_{m}(x)$ are the eigenfunctions of the interchange mode with mode number $m$. Moreover, one has $\beta=l_{\star} / w_{m}$ $\approx 0.5 \delta / w_{m}$, where $w_{m}$ is the maximal size of the island reached at the end of the quasilinear phase, $\delta$ is the radial width of the interchange band (IB) around the resonance, and $l_{\star}=L_{y} / m_{\star}$. The brackets $\langle.\rangle_{x}$ indicate a mean value in the IB, and the simple brackets $\langle$.$\rangle indicate a temporal mean$ on the nonlinear time scale $\tau_{N L}$, a characteristic time of the evolution of the large scales. Let us emphasize that Equation (5) describes the link between the pressure gradient in the island and the turbulence level. Indeed, the RHS of Eq. (5) is a measure of the intensity of the turbulence in the interchange band.

In order to test the validity of the model developed in Ref. 5, and, in particular, to test Eq. (5), we consider the run $\kappa_{2}=1.05$ and $\chi_{\perp}=3 \times 10^{-5}$ for which $w$ is of the order of a few $w_{c}^{\text {turb }}$. In Fig. 7, a zoom on the source term profile $\bar{S}_{\phi^{\prime}, p^{\prime}}(x)$ is shown for time $t=14522 \tau_{A}$. As argued in Ref. 5 where the model is derived, the source is well localized in the vicinity of the resonance $x=0$ in the $\mathrm{IB}$ (the $\mathrm{IB}$ is

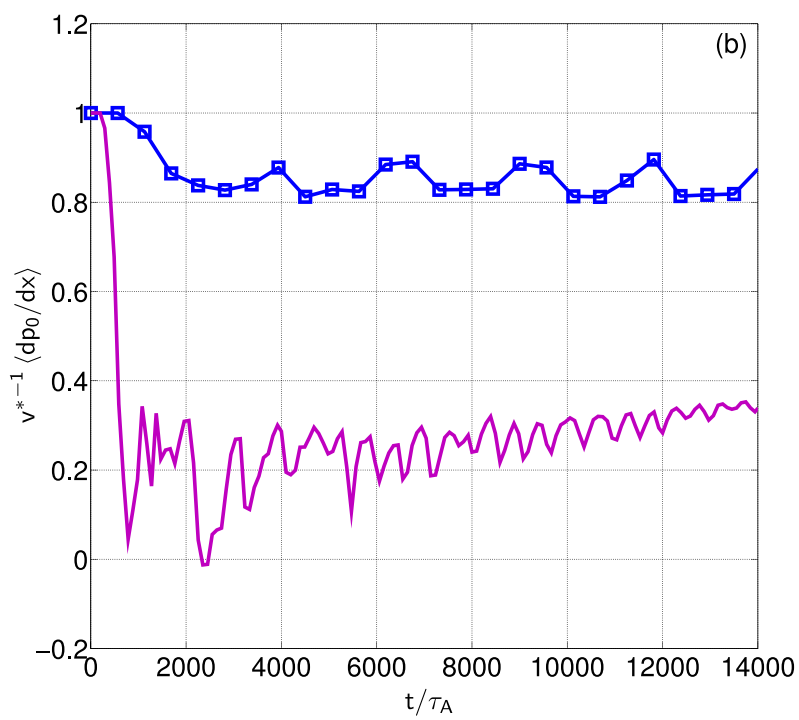

FIG. 6. For each color, the run parameters are the same in the left and right graphs of this figure and, also, in Fig. $1:$ (a) pressure profile at $t / \tau_{A}=5078$ (dashed line) and at the end of the nonlinear simulation $t / \tau_{A}=14037$ (full lines). (b) Time evolution of the gradient of the mean pressure profile into the island normalized to the equilibrium pressure gradient $v_{\star}$. 


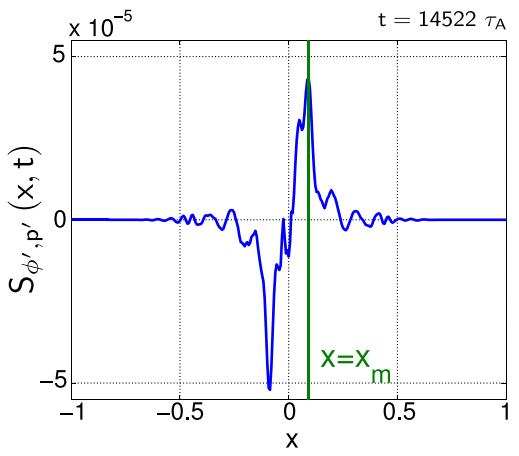

FIG. 7. $S_{\phi^{\prime}, p^{\prime}}(x, t)$ at $t=14522 \tau_{A}$ for the run $\kappa_{2}=1.05$ and $\chi_{\perp}=3 \times 10^{-5}$.

delimited by $|x| \leq \delta / 2 \approx 0.39)$ and has, predominantly, an odd parity. In order to give a self-consistent definition of means in Eq. (5), we explicitly set

$$
\left\langle\bar{S}_{\phi^{\prime}, p^{\prime}}\right\rangle_{x}(t)=\int_{0}^{x_{m}} d x \bar{S}_{\phi^{\prime}, p^{\prime}}(x, t),
$$

where $x_{m}$ is the position of the maximum of the source term in the IB. This choice allows us to follow the IB width fluctuations as shown in Fig. 7. Moreover, by definition,

$$
\langle f\rangle=\frac{1}{\tau_{N L}} \int_{t-\tau_{N L} / 2}^{t-\tau_{N L} / 2} f(t),
$$

where $\tau_{N L}=(d \ln w / d t)^{-1} \approx 980 \tau_{A}$ is computed in the quasilinear phase where the island width $w$ is almost a linear function of time. Let us emphasize that in the subsequent nonlinear phase, $\tau_{N L}$ also corresponds, roughly, to the characteristic time of oscillations of the large scale quantities. For instance, this behavior can be seen in Fig. 8 which shows the time evolution of the pressure gradient in the island $d P_{0} /$ $d x_{\text {island }}$ (the slope of the mean pressure in between the two radial extremities of the island).

Fig. 8 also shows the time evolution of the R.H.S of Eq. (5), with and without the temporal mean $\langle$.$\rangle . As$ expected, the temporal mean strongly smooths the curve. In agreement with the spectrum shown in Fig. 4 corresponding to this simulation, we have computed the R.H.S. by adding all the interchange mode contributions in Eq. (5) by taking $\Delta_{*}=15$ and $m_{\star}=35(m \in[20,50])$. In this graph, by definition, $\Gamma_{1}=\bar{S}_{\phi^{\prime}, p^{\prime}}(x, t)$ is the interchange contribution from the convective term in the mean pressure evolution equation. The other nonlinear contributions are neglected in this model and will be discussed below. It is found that the model predicts very well the time evolution of the mean pressure gradient into the island, including the nonlinear phase until $t \approx 5000 \tau_{A}$. The reason for which the model probably breaks down on large time scales is that it is not a dissipative model. To include dissipative effects, one should carefully model the energy cycle from interchange scales to large scales and the back transfer of this energy to interchange and dissipative scales. The latter mechanism is not included in the model and should decrease the value of the pressure gradient in the long time dynamics. However, the important point is that the model appears to be valid beyond the time where it reaches its maximal size. After this moment, the seed island growth is expected to be driven by neoclassical physics in tokamak devices. Thus, it becomes a neoclassical tearing mode which saturates on resistive time scales. This physics is not included in this model and is out of the scope of this paper. It is instructive to measure the robustness of this result by computing the same quantity for different $\Delta_{*}$. Two cases are presented in the left graph of Fig. 9. First, we consider the impact of the modes $m_{\star} \pm 1$, i.e., $\Delta_{*}=1$. It is found that the mean pressure gradient modification is strongly underestimated by the model. Second, for the case $\Delta_{*}=25$, the model overestimates in both the quasi-linear and nonlinear phase the gradient. The case $\Delta_{*}=32$ shows a result close but not as good as the case $\Delta_{*}=15$ and confirms the hypothesis that the quasi-adiabatic-modes do not give a net contribution to the generation of the mean pressure gradient. Note that it also shows that the modes are not fully adiabatic, otherwise $\Delta_{*}=32$ and $\Delta_{*}=25$ would give the same result. Finally, in Fig. 8, the impact on the model of the nonlinear term $\hat{\rho}^{2}\left\{\psi, \nabla_{\perp}^{2} \psi\right\}$ in the pressure equation evolution is shown. More precisely, we consider the interchange source term

$$
\Gamma_{2}=2 \hat{\rho}^{2} \sum_{\left|m-m_{*}\right| \leq \Delta_{*}, m>0} k_{m} \frac{d}{d x} \mathcal{I}\left(\hat{\psi}_{m} \hat{j}_{m}^{\star}\right) .
$$

The figure clearly shows that the contribution would be too large if one would take it into account in the model. There is in fact no reason to limit the sum of interchange modes for this nonlinearity, because there is no property equivalent to

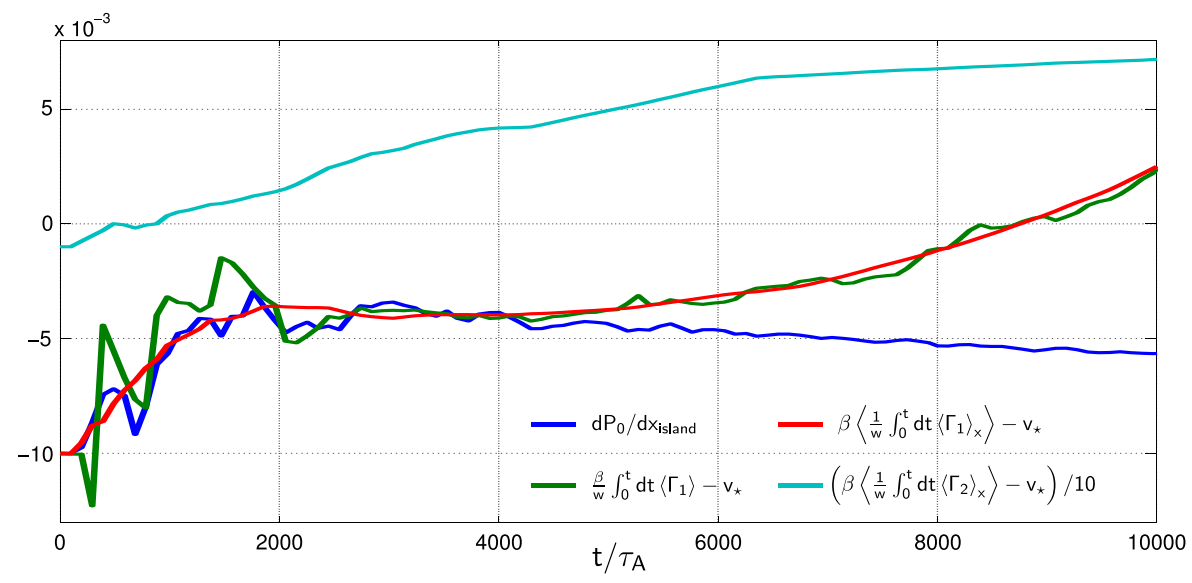

FIG. 8. Run $\kappa_{2}=1.05$ and $\chi_{\perp}=3 \times$ $10^{-5}$ : time evolution of the mean pressure gradient and comparison with the model Eq. (5). Interchange modes correspond to $\Delta_{*}=15$ and $m_{\star}=35$. 

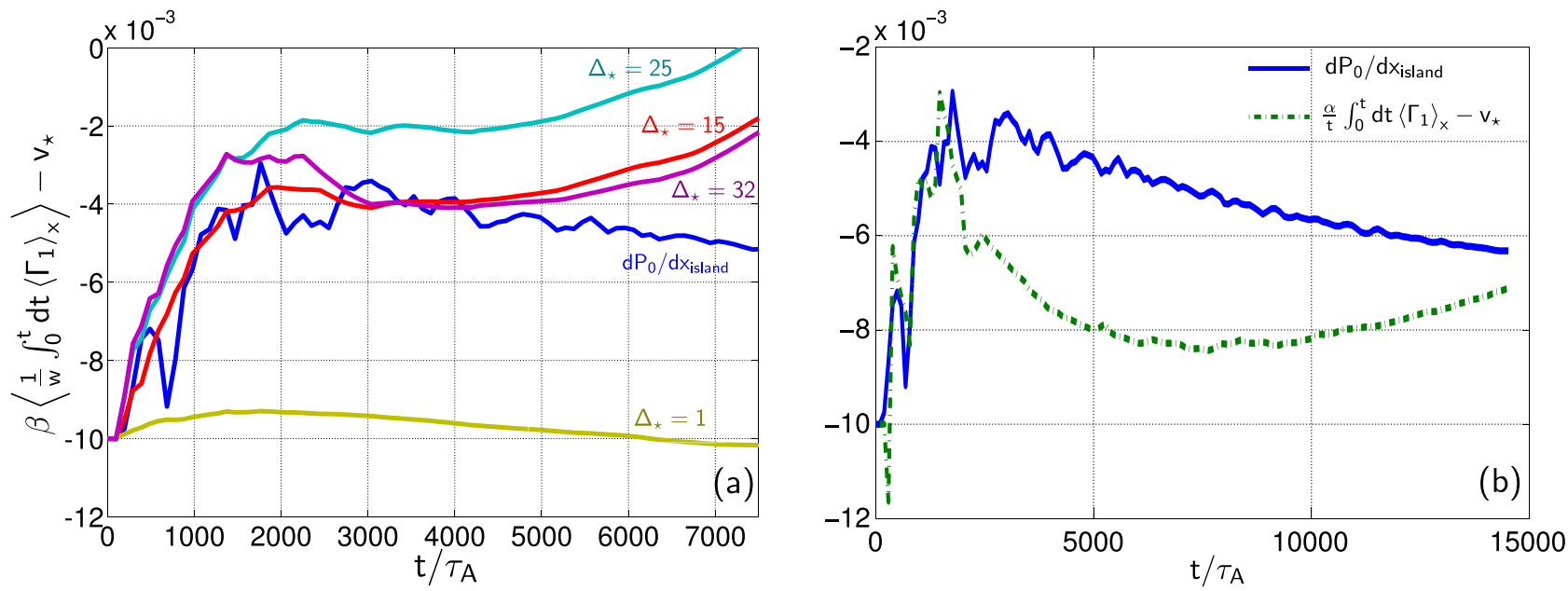

FIG. 9. Run $\kappa_{2}=1.05$ and $\chi_{\perp}=3 \times 10^{-5}$ : (a) time evolution of the mean pressure gradient and comparison with the model Eq. (9) with $\Delta_{*} \in\{15,1,25,32\}$. (b) Time evolution of the mean pressure gradient and comparison with the model Eq. (9), $\Delta_{*}=15$ and $m_{\star}=35$.

the quasi-adiabaticity. The point is that this term is almost canceled by the lower mode number contributions.

Eq. (5), coupled with the fact that in the quasi-linear phase the island size grows almost linearly with time, indicates that, in fact, the pressure flattening is linked to the mean energy transferred to large scales through the convective term. Indeed, introducing the ansatz $w(t)=\dot{w}_{\mathrm{ql}} t$, we obtain the following estimate:

$$
\nabla P_{0}^{\mathrm{isl}}(t)=-v_{\star}+\alpha\left\langle\frac{1}{t} \int_{0}^{t} d t\left\langle\Gamma_{1}\right\rangle_{x}\right\rangle,
$$

with $\alpha=2 \beta / \dot{w}_{\mathrm{ql}}$. The latter model is tested as shown in Fig. 9, without the mean on $\tau_{N L}$ time scales to make explicit the fluctuation level of $\left\langle\Gamma_{1}\right\rangle_{x}$. As just stated, we find that the model Eq. (9) works well in the quasilinear phase and a discrepancy between the model and the measure mean pressure gradient appears in the nonlinear phase. This is the most important phase. Indeed, it is at the end of the quasilinear phase that the seed island may reach its larger size and, thus, exceed the critical size $w_{c}^{\mathrm{ql}}$ to become an neoclassical tearing mode (NTM). We should point out that the nonlinear dynamics is characterized by multi-scale burst events. During these periods of characteristic duration, a fraction of $\tau_{N L}$, the island structure, and its width are not substantially affected. However, the source term $\bar{S}_{\phi^{\prime}, p^{\prime}}$ is not anymore localized in the IB as shown in Fig. 10 even though it has a reduced amplitude. In the long time dynamics, these phases disappear. For the reference run $\kappa_{2}=1.05, \chi_{\perp}=3 \times 10^{-5}$, and the last observed event occurs around $t=5700 \tau_{A}$. These events seem to occur after phases of pile up of energy at large scales and correspond to phases where there is a release of large scale energy to the smaller scales throughout the island and up to the dissipative scales. They are probably linked to the fact that interchange scales, by feeding island scales, tend to destabilize the island structure, which, in turn, relax quite abruptly the excess of energy in an intermittent and/or cyclical manner. The proof of this assertion and its study are out of the scope of this paper.

In experiments, pressure profile flattening of large neoclassical tearing modes can be observed despite the presence of the turbulence. For weak positive $\Delta^{\prime}$, in the long term dynamics, the flattening is also observed in our model ${ }^{23}$ despite the presence of turbulence. The validity of the present study is restricted to the seeding process of NTMs by turbulence, i.e., TDMIs. After the seeding by turbulence in the quasilinear phase, when the island is amplified, one may expect that some new mechanisms reduce the flattening. Indeed, in our interchange model, there is no amplification by neoclassical mechanisms and we do not take into account the multi-helical nature of the turbulence in devices. When the width of the island increases after the seeding, as shown in Ref. 3, on the long term, dynamics of the island also depends on the turbulence level outside the magnetic island. It follows that some other mechanisms may possibly reduce the role of the source-which imposes the partial character of the flattening ${ }^{5}$ - in the interchange band from where the island arises and therefore lead to a cancellation of the partial character of the flattening.

In our interchange model, the turbulence transport parameter is weakly reduced nonlinearly ${ }^{23}$ because the pressure gradient and therefore the turbulence level are reduced in the island. As the critical island width above which pressure flattening occurs is proportional to $\chi_{\perp}^{\text {turb }}{ }^{1 / 4} \propto \gamma_{\star}^{1 / 4}$, the
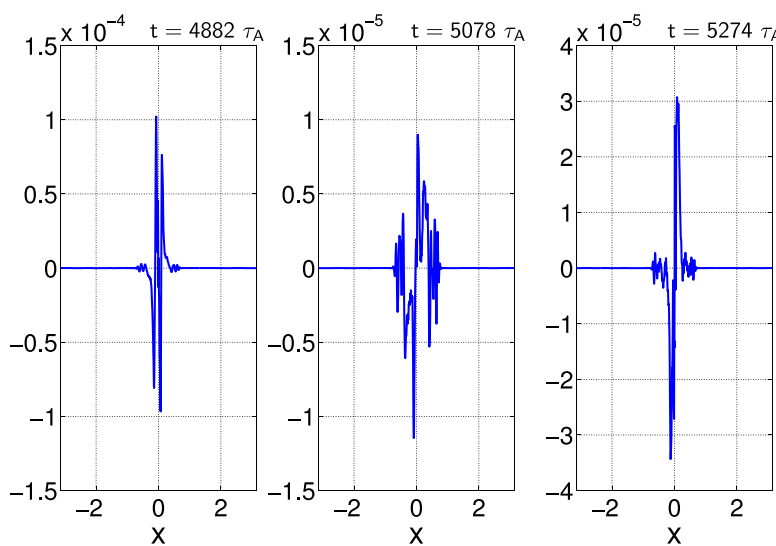

FIG. 10. Run $\kappa_{2}=1.05$ and $\chi_{\perp}=3 \times 10^{-5}: S_{\phi^{\prime}, p^{\prime}}(x, t)$ at the beginning of, during, and after a burst event. 
reduction in the turbulence level has almost no impact on the flattening process. The impact is stronger on the source $S \propto \gamma_{\star}$ in the interchange band, but one should have a decrease of at least one order of magnitude to observe an equilibration of the pressure into the island, which is not observed. Note that, the opposite situation, an enhancement of $\chi_{\perp}^{\text {turb }}$ by the turbulence in an island induced by a tearing instability, has been observed numerically for the ion temperature profile in a multi-helical electromagnetic model. ${ }^{25}$ For a stronger pressure flattening to emerge, the microturbulence level should be strongly reduced, for instance, by zonal flows which are underestimated in fluid models. A strong reduction in ion thermal diffusivity and therefore in the turbulence level in an island has already been measured in a magnetic island. ${ }^{14}$

\section{CONCLUSIONS}

We have pointed out the difficulty in studying experimentally the interaction between microturbulence and seed islands. A consequence of this is that the experimental detection of TDMI requires a signature to detect them. We have numerically investigated the generation of seed islands by small scale interchange modes in cases where they are characterized by a well established scale separation with MHD/ island physics and are localized in the vicinity of a resonant surface. We have shown that when the island size is significantly greater than the critical island width $w_{c}^{\text {turb }}$, the flattening occurs. We have shown that, when the ratio $w / w_{c}^{\text {turb }}$ is close to unity, TDMIs do not exhibit a pressure flattening. When the ratio is larger, it is dynamically correlated with it. We have also shown that the growth of TDMIs is self-driven by the presence of large scale quadripolar flow. It confers and confirms the ubiquitous character of these flows in reconnection problems. We have also shown that the pressure gradient inside a turbulence driven magnetic island can be explicitly linked to the properties of the interchange turbulence. All these results present a good agreement with the analytical model presented in Ref. 5. We have also shown that the nonlinear modification of the pressure gradient inside the island is proportional to the mean energy transferred to large scales through the convective term in the phase where the island grows.

As a future work, it would be interesting to validate this theory over a large parameter space and to extend it to multihelical turbulence induced by ion temperature gradient modes. Indeed, such a study would allow us to include specific turbulence scale lengths and their relationship with the seeded TDMIs. Nevertheless, this work indicates that a partial pressure profile flattening may not be linked to the size of the island $\left(w<w_{c}^{\text {turb }}\right)$ but to the fact that the seed island has been generated by turbulence. We have found that TDMI should be sensitive to the modulation of the turbulence into the islands on slow time scales compared with the turbulent time scales, i.e., MHD scales. This might give a signature if one wishes to detect them experimentally. Indeed, our results suggest that one may bring into light TDMIs in experiments by modulating the turbulence level inside magnetic islands.
One might observe a modification of the mean pressure gradient proportional to $w^{-1} \sqrt{E_{p^{\prime}} E_{k^{\prime}}}$, where $E_{p^{\prime}}$ and $E_{k^{\prime}}$ are, respectively, the kinetic and thermal energy of the turbulent bath.

\section{ACKNOWLEDGMENTS}

It is a pleasure to thank Dominique Escande for stimulating discussions. This work was granted access to the HPC resources of Aix-Marseille Université financed by the project Equip@Meso (ANR-10-EQPX-29-01) of the program "Investissements d'Avenir" supervised by the Agence Nationale pour la Recherche. Moreover, this research has been enabled by the use of computing resources provided by the Helios supercomputer in Japan and was supported in part by CNRS, AMU, CEA, and EURATOM.

${ }^{1}$ A. Ishizawa and N. Nakajuma, Phys. Plasmas 17, 072308 (2010).

${ }^{2}$ M. Muraglia, O. Agullo, S. Benkadda, M. Yagi, X. Garbet, and A. Sen, Phys. Rev. Lett. 107, 095003 (2011).

${ }^{3}$ A. Poyé, O. Agullo, M. Muraglia, X. Garbet, S. Benkadda, A. Sen, and N. Dubuit, Phys. Plasmas 22, 030704 (2015).

${ }^{4}$ W. A. Hornsby, P. Migliano, R. Buchholz, D. Zarzoso, F. J. Casson, E. Poli, and A. G. Peeters, Plasma Phys. Controlled Fusion 57, 054018 (2015).

${ }^{5}$ O. Agullo, M. Muraglia, S. Benkadda, A. Poyé, N. Dubuit, X. Garbet, and A. Sen, Phys. Plasmas 24, 042308 (2017).

${ }^{6}$ D. Raju, O. Sauter, and J. B. Lister, Plasma Phys. Controlled Fusion 45 , 369 (2003).

${ }^{7}$ G. Z. dos Santos Lima, Z. O. Guimaraes-Filho, A. M. Batista, I. L. Caldas, S. R. Lopes, R. L. Viana, I. C. Nascimento, and Yu. K. Kuznetsov, Phys. Plasmas 16, 042508 (2009).

${ }^{8}$ O. Sauter, M. A. Henderson, G. Ramponi, H. Zohm, and C. Zucca, Plasma Phys. Controlled Fusion 52, 025002 (2010).

${ }^{9}$ R. Fitzpatrick, Phys. Plasmas 2, 825 (1995).

${ }^{10}$ C. Yu, D. L. Brower, S. Zhao, R. V. Bravenec, J. Chen, H. Lin, N. C. Luhmann, Jr., W. A. Peebles, C. P. Ritz, P. M. Schoch, and X. Yang, Nucl. Fusion 32, 1545 (1992).

${ }^{11}$ P. C. de Vries, A. J. H. Donné, S. H. Heijnen, C. A. J. Hugenholtz, A. Krämer-Flecken, F. C. Schüller, and G. Waidmann, Nucl. Fusion 37, 1641 (1997).

${ }^{12}$ P. C. de Vries, G. Waidmann, A. Kramër-Flecken, A. J. H. Donné, and F. C. Schüller, Plasma Phys. Controlled Fusion 39, 439 (1997).

${ }^{13}$ J. A. Wesson, Plasma Phys. Controlled Fusion 37, A337 (1995).

${ }^{14}$ K. Ida, K. Kamiya, A. Isayama, Y. Sakamoto, and JT-60 Team, Phys. Rev. Lett. 109, 065001 (2012).

${ }^{15}$ K. Ida, T. Kobayashi, M. Yoshinuma, Y. Susuki, Y. Narushima, T. E. Evans, S. Tsuchiya, S. Inagaki, and K. Itoh, Nucl. Fusion 56, 092001 (2016).

${ }^{16}$ H. D. Stephens, D. J. Den Hartog, C. C. Hegna, and J. A. Reusch, Phys. Plasmas 17, 056115 (2010).

${ }^{17}$ L. Bardóczi, T. L. Rhodes, T. A. Carter, A. Banón Navarro, W. A. Peebles, F. Jenko, and G. McKee, Phys. Rev. Lett. 116, 215001 (2016).

${ }^{18}$ M. Muraglia, O. Agullo, S. Benkadda, X. Garbet, P. Beyer, and A. Sen, Phys. Rev. Lett. 103, 145001 (2009).

${ }^{19}$ M. Muraglia, O. Agullo, M. Yagi, S. Benkadda, P. Beyer, X. Garbet, S.-I. Itoh, K. Itoh, and A. Sen, Nucl. Fusion 49, 055016 (2009).

${ }^{20} \mathrm{~K}$. Takeda, O. Agullo, S. Benkadda, A. Sen, N. Bian, and X. Garbet, Phys. Plasmas 15, 022502 (2008).

${ }^{21}$ A. Poyé, O. Agullo, A. Smolyakov, S. Benkadda, and X. Garbet, Phys. Plasmas 20, 020702 (2013).

${ }^{22}$ A. Poyé, O. Agullo, A. Smolyakov, S. Benkadda, and X. Garbet, Phys. Plasmas 21, 020705 (2014).

${ }^{23}$ O. Agullo, M. Muraglia, A. Poyé, S. Benkadda, M. Yagi, X. Garbet, and A. Sen, Phys. Plasmas 21, 092303 (2014).

${ }^{24}$ D. F. Escande and M. Ottaviani, Phys. Lett. A 323, 278 (2004).

${ }^{25} \mathrm{~J}$. Li, Y. Kishimoto, and Z. X. Wangt, Phys. Plasmas 21, 020703 (2014). 